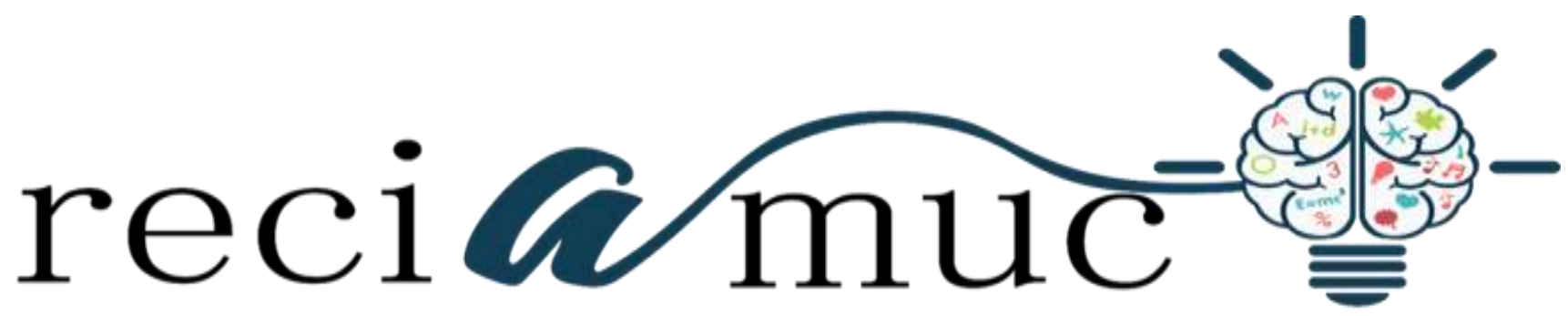

Revista científica de investigación actualización del mundo de las ciencias

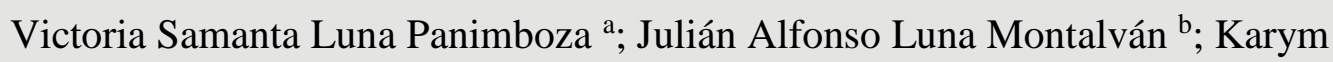
Gabriela Jácome Vera ${ }^{c}$; Jorge Erwin Yagual Hidalgo ${ }^{\text {d }}$

Complicaciones anestésicas en la cirugía laparoscópica pediátrica y manejo entre el periodo de julio del 2018 a abril del 2019

Anesthetic complications in pediatric laparoscopic surgery and management between the period from July 2018 to April 2019

Revista Científica de Investigación actualización del mundo de las Ciencias. Vol. 3 núm., 2, abril, ISSN: 2588-0748, 2018, pp. 143-154

DOI: $10.26820 /$ reciamuc/3.(2).abril.2019.143-154

URL: $\underline{\text { http://reciamuc.com/index.php/RECIAMUC/article/view/409 }}$

Código UNESCO: 3205 Medicina Interna

Tipo de Investigación: Artículo de Investigación

Editorial Saberes del Conocimiento

Recibido: 21/02/2019 Aceptado: 10/03/2019 Publicado: 30/04/2019

Correspondencia: victoria_luna19@outlook.es

a. Médico General; Hospital General Norte de Guayaquil Los Ceibos. Guayaquil - Ecuador; victoria_luna19@outlook.es

b. Médico especialista en cirugía pediátrica; Hospital General Norte de Guayaquil Los Ceibos. Guayaquil Ecuador; d.rjluna@ hotmail.com

c. Médico General; Hospital General Norte de Guayaquil Los Ceibos. Guayaquil - Ecuador; kgjv@gmail.com

d. Médico General; Hospital General Norte de Guayaquil Los Ceibos. Guayaquil - Ecuador; jorge40.jy@gmail.com 


\section{Complicaciones anestésicas en la cirugía laparoscópica pediátrica y manejo entre el periodo de julio del 2018 a abril del 2019}

Vol. 3, núm. 2., (2019)

Victoria Samanta Luna Panimboza; Julián Alfonso Luna Montalván; Karym Gabriela Jácome Vera; Jorge Erwin Yagual Hidalgo

\section{RESUMEN}

Objetivos: Evaluar el manejo y comportamiento de los anestésicos durante la cirugía laparoscópica en niños menores de 15 años durante 10 meses. Conocer complicaciones que se producen posterior a la administración de los anestésicos. Método: Se realizó un estudio longitudinal prospectivo descriptivo con el objetivo de conocer el efecto de los anestésicos durante una cirugía laparoscópica en niños menores de 15 años desde Julio del 2018 hasta abril del 2019 que equivale a 10 meses. La muestra constituyó de 209 casos. Se evaluó en las diferentes etapas que conforman el tiempo anestésico y las complicaciones que presentaron los pacientes. Resultados: El propofol, fentanilo y rocuronio fueron los medicamentos más usados durante la inducción anestésica y el sevofluorane para el mantenimiento. El broncoespasmo es la principal complicación anestésica. Conclusiones: La mayoría de los pacientes evolucionaron satisfactoriamente.

Palabras Claves: Cirugía laparoscópica pediátrica; anestesia pediátrica. 


\title{
Complicaciones anestésicas en la cirugía laparoscópica pediátrica y manejo entre el periodo de julio del 2018 a abril del 2019
}

Vol. 3, núm. 2., (2019)

Victoria Samanta Luna Panimboza; Julián Alfonso Luna Montalván; Karym Gabriela Jácome Vera; Jorge Erwin Yagual Hidalgo

\begin{abstract}
Objectives: To evaluate the management and behavior of anesthetics during laparoscopic surgery in children under 15 years of age for 10 months. Know complications that occur after the administration of anesthetics. Method: A prospective descriptive longitudinal study was carried out in order to know the effect of anesthetics during laparoscopic surgery in children under 15 years of age from July 2018 to April 2019, which is equivalent to 10 months. The sample constituted 209 cases. It was evaluated in the different stages that make up the anesthetic time and the complications that patients presented. Results: Propofol, fentanyl and rocuronium were the drugs most used during anesthetic induction and sevofluorane for maintenance. Bronchospasm is the main anesthetic complication. Conclusions: The majority of patients progressed satisfactorily.
\end{abstract}

Key Words: Pediatric laparoscopic surgery; pediatric anesthesia. 


\section{Complicaciones anestésicas en la cirugía laparoscópica pediátrica y manejo entre el periodo de julio del 2018 a abril del 2019}

Vol. 3, núm. 2., (2019)

Victoria Samanta Luna Panimboza; Julián Alfonso Luna Montalván; Karym Gabriela Jácome Vera; Jorge Erwin Yagual Hidalgo

\section{Introducción.}

La laparoscopía ha estado disponible desde 1923, pero solo en las últimas décadas ha sido aplicada en cirugía pediátrica, mostrando varias ventajas sobre las técnicas convencionales.

Según la revista New England Journal of Medicine, la cirugía laparoscópica fue uno de los cinco grandes campos de progreso de la cirugía pediátrica en la última década del Siglo XX.

La cirugía laparoscópica ha producido una revolución quirúrgica muy significativa en la medicina moderna; el espectro de la cirugía laparoscópica se ha extendido desde la simple cirugía abdominal, hasta la cirugía torácica compleja; de esta manera, se ha convertido en una especialidad desafiante para la Anestesiología.

El manejo laparoscópico ofrece muchas ventajas: reduce potencialmente el estrés quirúrgico y las pérdidas de fluidos que lo acompañan; hay menor necesidad de analgesia postoperatoria; reduce las complicaciones respiratorias, de la herida, y el tiempo de convalecencia postoperatoria, inclusive cuando se requiere cuidados intensivos; permite un rápido regreso a la dieta normal y disminuye el tiempo total de hospitalización. Las desventajas principales incluyen mayor tiempo quirúrgico y mayor incidencia de abscesos intraabdominales. El neumoperitoneo creado y las posiciones extremas adoptadas suponen un reto en el manejo anestésico, debido a los cambios fisiológicos inducidos por estos dos. La elección de la técnica anestésica en niños sometidos a laparoscopía se debe considerar la función cardiopulmonar subyacente, realizando las modificaciones necesarias para permitir que estas operaciones se realicen de forma segura, en especial cuando se realizan en pacientes con patologías previas y así 


\section{Complicaciones anestésicas en la cirugía laparoscópica pediátrica y manejo entre el periodo de julio del 2018 a abril del 2019}

Vol. 3, núm. 2., (2019)

Victoria Samanta Luna Panimboza; Julián Alfonso Luna Montalván; Karym Gabriela Jácome Vera; Jorge Erwin Yagual Hidalgo

vigilar con más atención la aparición de complicaciones intraoperatorias para resolverlas oportunamente.

Idealmente se usará un dispositivo de la vía aérea, libre de fugas durante el neumoperitoneo; los tubos endotraqueales con balón, cuando es posible, permiten una adecuada ventilación pulmonar. Mantener una adecuada relajación muscular y tomar medidas preventivas contra la hipotermia son puntos clave. El manejo del dolor se debe realizar en base a una estrategia multimodal.

La técnica anestésica a emplear dependerá de la experiencia del anestesiólogo, el tipo de intervención y siempre tomando en cuenta los cambios fisiopatológicos que se producen durante la introducción de $\mathrm{CO}_{2}$ para realización del neumoperitoneo. Para la colecistectomía laparoscópica, por ejemplo, la mayoría de los anestesiólogos prefiere la anestesia general inhalatoria con ventilación controlada, de manera tal que si aumenta la presión de $\mathrm{CO}_{2}$ respiratoria, se pueda hiperventilar al paciente para barrer el $\mathrm{CO}_{2}$.

La anestesia general con intubación y ventilación controlada previenen el riesgo de hipoventilación y regurgitación ligada a la presión intradominal incrementada y a la postura.

La ventilación controlada limita las perturbaciones cardiorrespiratorias ligadas a la laparoscopia. Un FiO2 de 0,3 a 0,5 permite la prevención de hipoxia.

Una hiperventilación moderada (volúmenes tidales mayores y/o aumento de la frecuencia respiratoria en $50 \%$ superior a las necesidades básicas) previene la hipercapnea. Los anestésicos 


\section{Complicaciones anestésicas en la cirugía laparoscópica pediátrica y manejo entre el periodo de julio del 2018 a abril del 2019}

Vol. 3, núm. 2., (2019)

Victoria Samanta Luna Panimboza; Julián Alfonso Luna Montalván; Karym Gabriela Jácome Vera; Jorge Erwin Yagual Hidalgo

con acción básico dilatadora pueden ser utilizados para minimizar los efectos hemodinámicos, así como también evitar los agentes depresores miocárdicos.

Durante la anestesia en cirugía laparoscópica vamos a encontrar una serie de cambios fisiopatológicos que dependerán de la insuflación de CO2 dentro de la cavidad abdominal, produciéndose alteraciones hemodinámicas, respiratorias, metabólicas y en otros sistemas.

Referente a las drogas inductoras, no existe alguna preferencia, ya que se puede utilizar el tiopental sódico como el propofol, siempre y cuando no exista contraindicación para alguno de ellos. Se puede utilizar relajantes musculares de acción intermedia y corta, como el bromuro de vecuronio, besilato de atracurio o bromuro de rocuronio. Sin embargo, hay que tener cuidado con el atracurio, por desencadenar liberación de histamina. Si se desea usar opiáceos, se debe usar los de acción corta, como el alfentanyl. Para el mantenimiento de la anestesia, se puede utilizar los halogenados recomendables para la cirugía ambulatoria, como el isoflurano, sevoflurano o desflurano. Hay que tener en cuenta que una vez se intube al paciente colocar una sonda nasogástrica para descomprimir el estómago, pues muchas veces la distensión gástrica dificulta visualizar las vísceras abdominales.

En relación al óxido nitroso (N2O), su uso en laparoscopia sigue siendo muy controversial, aunque no está contraindicado. Se dice que como es más difusible tenderá a aumentar la presión abdominal; otros lo relacionan con la persistencia del dolor en el postoperatorio. En la medida de lo posible, se tenderá a realizar el mantenimiento de la anestesia con oxígeno y aire para evitar complicaciones. 


\section{Complicaciones anestésicas en la cirugía laparoscópica pediátrica y manejo entre el periodo de julio del 2018 a abril del 2019}

Vol. 3, núm. 2., (2019)

Victoria Samanta Luna Panimboza; Julián Alfonso Luna Montalván; Karym Gabriela Jácome Vera; Jorge Erwin Yagual Hidalgo

Al finalizar la cirugía laparoscópica la exsuflación del neumoperitoneo debe ser lenta; un aflujo brutal de sangre rica en metabolitos hacia la circulación central, realiza una verdadera embolia ácida. El retorno a la posición horizontal debe ser progresivo para asegurar reperfusión homogénea y prevenir hipotensión. Se debe buscar el retorno progresivo a una situación hemodinámica y ventilación anteriores. La prevención y tratamiento de morbilidad postoperatoria (dolor, náuseas, vómitos), deben ser tenidos en cuenta. Se recomienda metoclorpropamida, diclofenaco, poco antes del fin de la laparoscopia.

Por todas estas razones resulta oportuno desarrollar un estudio que permita utilizar de manera eficaz los resultados obtenidos, para obtener mejores beneficios anestésicos en la cirugía laparoscópica pediátrica, de manera que se estandarice el manejo de estos pacientes con la consiguiente extensión de esta técnica quirúrgica a mayor número de entidades nosológicas.

\section{Materiales y métodos.}

Se realizó un estudio longitudinal prospectivo descriptivo con el objetivo de conocer el efecto de los anestésicos durante la cirugía laparoscópica en niños menores de 15 años desde Julio del 2018 hasta abril del 2019. Se envía una solicitud a Docencia del Hospital en estudio para que nos faciliten los datos correspondientes de los pacientes con los CIE de interés donde excluimos procedimientos quirúrgicos no laparoscópicos y mayores de 15 años.

La fuente primaria de la investigación se basa en las historias clínicas de cada paciente. La cuantificación de los datos se realizó en una plantilla Microsoft Excel 2010. Los datos fueron procesados y los resultados estadísticos demostrados en gráficos y tablas. 


\section{Complicaciones anestésicas en la cirugía laparoscópica pediátrica y manejo entre el periodo de julio del 2018 a abril del 2019}

Vol. 3, núm. 2., (2019)

Victoria Samanta Luna Panimboza; Julián Alfonso Luna Montalván; Karym Gabriela Jácome Vera; Jorge Erwin Yagual Hidalgo

\section{Resultados.}

El sexo que predomina en el estudio es el sexo masculino (62\%) en comparación con el sexo femenino (38\%). (Tabla I).

\begin{tabular}{lll}
\hline SEXO & CANTIDAD & $\%$ \\
Femenino & 79 & 38 \\
Masculino & 130 & 62 \\
TOTAL & 209 & 100 \\
\hline
\end{tabular}

Tabla I. Distribución de los pacientes de acuerdo al sexo

La edad que más prevalece fue niños de 11 años (16.3\%), 9 años (11\%) y 14 años (12.9\%). La mayoría de los pacientes en un $92 \%$ no presentaron ninguna enfermedad asociada a diferencia de otras como asma (62\%), y cardiopatías (1\%) (Tabla II).

\begin{tabular}{lll}
\hline ENFERMEDADES & CANTIDAD & $\%$ \\
ASOCIADAS & & \\
Asma & 13 & 62 \\
Púrpura & 1 & 0.5 \\
Trombocitopénica & & \\
Cardiopatía & 3 & 1 \\
Rinitis & 2 & 1 \\
Ninguna & 193 & 92 \\
TOTAL & 209 & 100 \\
\hline
\end{tabular}

Tabla II. Distribución de los pacientes de acuerdo a las enfermedades asociadas

En relación con el método anestésico, la anestesia general balanceada fue aplicada sobre 206 pacientes $(99 \%)$ a diferencia de la anestesia endovenosa (1\%). En cuanto a la medicación 


\section{Complicaciones anestésicas en la cirugía laparoscópica pediátrica y manejo entre el periodo de julio del 2018 a abril del 2019}

Vol. 3, núm. 2., (2019)

Victoria Samanta Luna Panimboza; Julián Alfonso Luna Montalván; Karym Gabriela Jácome Vera; Jorge Erwin Yagual Hidalgo

administrada durante las diferentes etapas de la anestesia tenemos: en la premedicación fue el midazolam en $1 \%$ del total de los pacientes, la mayoría no le administraron medicación durante esta etapa de la anestesia. Como observamos en la tabla III, durante la inducción a 204 pacientes usaron propofol (98\%), se les administró como relajante muscular el rocuronio en 204 pacientes (98\%), fentanilo en 179 pacientes (86\%), lidocaína al 2\% sin epinefrina en 134 pacientes (64\%), usaron midazolan en 99 pacientes (47\%), sevofluorane en 20 pacientes (10\%) y remifentanilo en 7 pacientes $(3 \%)$. (Tabla III).

\begin{tabular}{lll}
\hline INDUCCIÓN & CANTIDAD & $\%$ \\
ANESTÉSICA & & \\
Midazolam & 99 & 47 \\
Propofol & 204 & 98 \\
Lidocaína 2\% S/E & 134 & 64 \\
Fentanilo & 179 & 86 \\
Rocuronio & 204 & 98 \\
Sevofluorane & 20 & 10 \\
Remifentanilo & 7 & 3 \\
\hline
\end{tabular}

Tabla III. Distribución de los pacientes de acuerdo a la inducción anestésica

Durante el mantenimiento anestésico refleja que 207 pacientes usaron sevofluorane (99\%) tal como lo demuestra la Tabla IV.

\begin{tabular}{lll}
\hline MANTENIMIENTO & CANTIDAD & $\%$ \\
ANESTÉSICO & & \\
Sevofluorane & 207 & 99 \\
Propofol & 2 & 1 \\
Remifentanilo & 33 & 16 \\
\hline
\end{tabular}

Tabla IV. Distribución de los pacientes de acuerdo al mantenimiento anestésico 


\section{Complicaciones anestésicas en la cirugía laparoscópica pediátrica y manejo entre el periodo de julio del 2018 a abril del 2019}

Vol. 3, núm. 2., (2019)

Victoria Samanta Luna Panimboza; Julián Alfonso Luna Montalván; Karym Gabriela Jácome Vera; Jorge Erwin Yagual Hidalgo

Al finalizar el procedimiento anestésico se realiza la reversión con un inhibidor de la acetilcolinesterasa como la neostigmina en conjunto con la atropina en 88 pacientes, a 3 pacientes se los revertió con suganmadex y en la mayoría no se usó ninguna medicación $(56.5 \%)$.

Entre las complicaciones anestésicas como muestra la tabla $\mathrm{V}$ presentamos al broncoespasmo en un $6 \%$, relajación persistente en un $1 \%$, rash cutáneo en un paciente y en gran porcentaje (92\%) no presentaron ninguna complicación. En cuanto a las complicaciones presentadas en postquirúrgico sólo el dolor en 3 pacientes.

\begin{tabular}{lll}
\hline COMPLICACIONES & CANTIDAD & $\%$ \\
ANESTÉSICAS & & \\
Broncoespasmo & 12 & 6 \\
Relajación persistente & 3 & 1 \\
Rash cutáneo & 1 & 1 \\
Ninguno & 193 & 92 \\
TOTAL & 209 & 100 \\
\hline
\end{tabular}

Tabla V. Distribución de los pacientes de acuerdo a las complicaciones anestésicas

\section{Conclusiones.}

La mayor parte de cirugías laparoscópicas se desarrolló en un $62 \%$ en el sexo masculino y fueron intervenidos quirúrgicamente para apendicectomia en un $78 \%$.

El método anestésico más utilizado fue la anestesia general combinada. 


\section{Complicaciones anestésicas en la cirugía laparoscópica pediátrica y manejo entre el periodo de julio del 2018 a abril del 2019}

Vol. 3, núm. 2., (2019)

Victoria Samanta Luna Panimboza; Julián Alfonso Luna Montalván; Karym Gabriela Jácome Vera; Jorge Erwin Yagual Hidalgo

En su mayoría fueron inducidos por propofol (98\%), fentanilo $(86 \%)$ y rocuronio en intubación orotraqueal (98\%) y mantenidos con sevofluorane en un $99 \%$.

En gran porcentaje respondieron a la reversión clásica (atropina mas neostigmina).

La principal complicación fue el broncoespasmo, este se da tanto en el transquirúrgica como a la extubación del paciente en un $6 \%$.

La principal complicación postquirúrgica fue el dolor (1\%), el cual fue controlado gracias a la analgesia transquirúrgica.

\section{Bibliografía.}

1. Nápoles SR, Oliva RM. Anestesia videoendoscópica en el niño. Medisan. 2012;16(1):29-34.

2. Echemendía - Acosta I., Sanchez G., De la Paz C., Pozo J. Comportamiento anestésico en la cirugía laparoscópica pediátrica. Medigraphic. Vol 41. Julio - septiembre 2018; 183-195

3. Gómez PB, García RR, López MS, Triana M. Comportamiento ventilatorio- hemodinámico introaperatorio en la colecistectomía laparoscópica pediátrica. Rev Cub Anestesiol Reanim [Internet]. 2002 [citado 1 Mar 2017]; 1(1): [aprox. 8 p]. Disponible en: http://bvs.sld. cu/revistas/scar/vol1/scar010302.pdf.

4. Laverde Sabogal CE, Betancur VivasD. Cardiac arrest during laparoscopic cholecystectomy. Rev Colomb Anestesiol [Internet]. 2013 [citado 1 Mar 2017]; 41(4): [aprox. 4 p]. Available in: http://www. sciencedirect.com/science/article/pii/S2256208713000618.

5. Treviño CJ, Galván MA, Landa GR, Moreno M, Palacios RJ, DíazPizarro JI y cols. Cirugía endoscópica pediátrica. Experiencia inicial en un hospital general. Asociación Mexicana de Cirugía Endoscópica, AC [Internet]. 2002 Ene-Mar [citado 13 Feb 2017]; 3(1): [aprox. 4 p]. Disponible en: http://www.medigraphic.com/pdfs/endosco/ce-2002/ ce021e.pdf.

6. Gonzálvez BJ, Paco LH, Cabrera SJ, Puente FC. Caracterización de pacientes operados mediante colecistectomía laparoscópica en un hospital pediátrico. Medisan [Internet]. 2015 [citado 20 Feb 2017]; 19(4): [aprox. 10 p]. Disponible en: http://scielo.sld.cu/scielo.php?script=sci_ar ttext\&pid=S1029-30192015000400004. 


\section{Complicaciones anestésicas en la cirugía laparoscópica pediátrica y manejo entre el periodo de julio del 2018 a abril del 2019}

Vol. 3, núm. 2., (2019)

Victoria Samanta Luna Panimboza; Julián Alfonso Luna Montalván; Karym Gabriela Jácome Vera; Jorge Erwin Yagual Hidalgo

7. Salazar VM, González RP, Luzardo SE, Rodríguez RR, Puertas AJ. Cirugía mayor ambulatoria en pacientes operados de urgencia. MEDISAN [Internet]. 2013 [citado 1 Mar 2017]; 17(6): [aprox. 2 p]. Disponible en: http://scielo.sld.cu/pdf/san/v17n6/san10176.pdf.

8. Gómez LM, Ocampo F, Andrés Orozco J, Caicedo J. Effi cacy of anesthetic premedication in pediatric patients using oral midazolam and acetaminophen. Observational study. Rev Colomb Anestesiol [Internet]. 2013 [citado 12 Feb 2017];41(1):[aprox. 5 p]. Available in: http://www. sciencedirect.com/science/article/pii/S2256208712000454.

9. Fuentes S, Cano I, López M, García A, Morante R, Moreno C. Cambios cardiovasculares y ventilatorios durante la laparoscopía en neonatos y lactantes pequeños Hospital Universitario 12 de Octubre. Madrid Cir Pediatr [Internet]. 2012 [citado 25 Ene 2017]; 25(3):[aprox. 3 p]. Disponible en: http://www.secipe.org/coldata/upload/revista/2012_25-3_126-128.pdf.

10. Enciso NJ. Anestesia en la cirugía laparoscópica abdominal. An Fac Med. Scielo. Enero 2013;74:63-70.

11. García D, Ramirez L, Hernández D.Consideraciones anestésicas en cirugía laparoscópica en el paciente pediátrico. Revista Mexicana de anestesiología. Medigraphic. Vol 35. Abril Junio 2012; 164-167. 